A.A. Korobko

\title{
MULTIFREQUENCY ALGORITHMS FOR DETERMINING THE MOISTURE CONTENT OF LIQUID EMULSIONS BY THE METHOD OF RESONANCE DIELCOMETRY
}

Purpose. The main attention is paid to the development and investigation of multifrequency algorithms for the realization of the method of resonance dielcometric measurement of the humidity of emulsions of the type "nonpolar liquid dielectric-water». Multifrequency algorithms take into account the problem of «uncertainty of varieties» and increase the sensitivity of the dielcometric method. Methodology. Multifrequency algorithms are proposed to solve the problem of «uncertainty of varieties» and improve the metrological characteristics of the resonance dielcometric method. The essence of the algorithms is to use a mathematical model of the emulsion and to determine the permittivity of the dehydrated liquid and the emulsion. The task of developing algorithms is to determine and take into account the influence of the parasitic electrical capacitance of the measuring oscillator and the measuring transducer. The essence of the method consists in alternately determining the resonance frequency of the oscillatory circuit with various configurations, which allows to take into account errors from parasitic parameters. The problem of "uncertainty of varieties" is formulated and solved. The metrological characteristics of the resonance dielcometric method are determined using algorithms. Results. Frequency domains of application of mathematical model of an emulsion are defined. An algorithm in a general form with four frequencies suitable for practical implementation in dielcometric resonance measurements is developed. Partial algorithms with three and two frequencies are developed. The systematic values of simulation errors in the emulsion in the microwave range are determined. Generalized metrological characteristics are obtained. The ways of increasing the sensitivity of the dielcometric method are determined. The problem of «uncertainty of varieties" was solved. Experimental data on determination of humidity for the developed algorithms are obtained. The value of the volumetric moisture in the transformer oil was measured. Originality. New multifrequency algorithms for determining the moisture content by the resonance dielcometric method have been proposed, investigated and practically realized. A generalized metrological characteristic for an algorithm with four frequencies is obtained. Metrological characteristics of algorithms for three and two frequencies are obtained. The problem of «uncertainty of varieties» was solved. Recommendations for increasing the sensitivity of dielcometric resonance moisture meters are developed and implemented. Practical value. The results of this work allow to solve the problem of "variability of varieties", increase sensitivity and accurately determine the moisture content in most nonpolar liquid dielectrics to a value of $10^{-5}$. This is applicable in a large field of electrical engineering, machine building, oil refining and the chemical industry. References 10, tables 3, figures 2.

Key words: moisture, method of resonance dielcometry, varietal uncertainty, mathematical model of emulsion, multifrequency algorithm, generalized metrological characteristics.

Предложсены многочастотные алгоритмы определения влагосодержсания эмульсии типа: «неполярный жсидкий диэлектрик - вода» методом резонансной диэлькометрии. Использована математическая модель эмульсии с представлением воды в виде идеально проводящих сфер. Определены частотные области применимости модели в ВЧ и СВЧ диапазонах. В СВЧ диапазоне определены величины систематических погрешностей модели. Для решения проблемы «сортовой неопределенности" $и$ улучшения метрологических характеристик резонансного диэлькометрического метода разработан четырехчастотный алгоритм, являющийся обобщенным видом трех- и двухчастотных алгоритмов. Определена обобщенная метрологическая характеристика четырехчастотного алгоритма и его предельные характеристики. Получены метрологические характеристики для трех- и двухчастотных алгоритмов. Проведен анализ погрешностей и корректности теоретических исследований путем сравнения их результатов с экспериментальными данными для ВЧ и СВЧ диапазонов. Библ. 10, табл.3, рис.2.

Ключевые слова: влагосодержание, резонансная диэлькометрия, сортовая неопределенность, математическая модель эмульсии, многочастотный алгоритм, метрологическая характеристика.

Introduction. The problem of determining the moisture content of technical fluids is relevant for practical applications in various areas of industry. In this case, the lower values of the measured values of the volumetric moisture content $\mathrm{W}$ in emulsions of the «nonpolar liquid dielectric-water» type can be from 10 $\mathrm{cm}^{3} / \mathrm{m}^{3}$ for transformer oil up to $100 \mathrm{~cm}^{3} / \mathrm{m}^{3}$ for aviation kerosene. The measurement of such small values of moisture content is a complex technical task and requires the development of methods and technical means for measuring moisture content with the appropriate characteristics.
Using dielcometry for the calculated determination of the volume moisture content $W$ of emulsions is based on the dependence of the dielectric constant $\varepsilon_{e m l}$ of a homogeneous emulsion of the «liquid nonpolar dielectricwater» type on the moisture content and permittivity $\varepsilon_{n l d}$ of an anhydrous nonpolar liquid dielectric. Moreover, this dependence can be obtained both experimentally (by the method of preliminary calibration of moisture meters using «reference» emulsions) and analytically, in the form of a functional connection, the values of the volume moisture content $W$ and the dielectric permittivities $\varepsilon_{e m l}$

(C) A.A. Korobko 
and $\varepsilon_{n l d}$. This functional relationship is determined on the basis of various mathematical models of the emulsion. In both cases, the reliability of the results obtained is significantly influenced by the so-called «varietal uncertainty». This is the dependence of the moisture content on the magnitude of the permittivity and, consequently, on the grade of the liquid being investigated.

In this paper, theoretical and practical aspects of ways to improve metrological characteristics, both the dieelcometric method itself and technical means for measuring the moisture content of nonpolar liquids, are simultaneously considered, while solving the problem of «varietal uncertainty».

Analysis of the current state of the problem. To determine low levels of volumetric moisture content, a high sensitivity of the method is necessary. The maximum sensitivity is characterized by resonance dielcometric methods. The essence of these methods is to determine the dielectric constant of a liquid by measuring the resonance frequency of an oscillating circuit, which includes a capacitive type measuring transducer (MT) filled with the test liquid. They use a wide range of frequencies of electromagnetic oscillations from sound to $\mathrm{HF}$ and microwave ranges [1].

In order to clarify the technical level of modern resonant high-frequency and microwave dielcometric moisture meters for liquid nonpolar dielectrics, an analysis of the characteristics of modern standard moisture meters on the market was carried out (Table 1).

The moisture meters considered differ substantially in their technical level, which can be characterized by the magnitude of the absolute error in measuring the moisture content at the lower range.

Table 1

Characteristics of typical modern HF and microwave moisture meters

\begin{tabular}{|c|c|c|}
\hline No. & $\begin{array}{c}\text { Moisture meter type } \\
\text { (measurement range } W, \mathrm{~cm}^{3} / \mathrm{m}^{3} \text { ) }\end{array}$ & $\begin{array}{l}\text { Absolute measurement } \\
\text { error, } \mathrm{cm}^{3} / \mathrm{m}^{3}\end{array}$ \\
\hline 1 & $\begin{array}{l}\text { EASZ-1 } \\
\left(0-10^{4}\right)\end{array}$ & \pm 100 \\
\hline 2 & $\begin{array}{l}\text { УВДН-1ПМ1 } \\
\left(100-6 \cdot 10^{4}\right)\end{array}$ & \pm 100 \\
\hline 3 & $\begin{array}{l}\text { EASZ-1BS\&W } \\
\left(0-10^{4}\right)\end{array}$ & \pm 100 \\
\hline 4 & $\begin{array}{l}\text { FIZEPR - SW 100.30 } \\
(1000-3000)\end{array}$ & \pm 200 \\
\hline 5 & $\begin{array}{l}\text { ЭУДВН-1л } \\
\left(200-2 \cdot 10^{4}\right) \\
\end{array}$ & \pm 200 \\
\hline 6 & $\begin{array}{c}\text { УДВН-1эп } \\
\left(100-2 \cdot 10^{4}\right) \\
\end{array}$ & \pm 250 \\
\hline 7 & $\begin{array}{l}\text { Universal IV CM } \\
\left(0-10^{4}\right)\end{array}$ & \pm 300 \\
\hline 8 & $\begin{array}{l}\text { OW-301 } \\
\left(0-10^{4}\right) \\
\end{array}$ & \pm 500 \\
\hline 9 & $\begin{array}{l}\mathrm{MBH}-1 \\
\left(0-3 \cdot 10^{4}\right) \\
\end{array}$ & \pm 500 \\
\hline 10 & $\begin{array}{l}\text { PHASE DYNAMICS - L } \\
\qquad\left(0-2 \cdot 10^{4}\right)\end{array}$ & \pm 500 \\
\hline
\end{tabular}

As can be seen from Table 1, the dielcometric hygrometers corresponding to the best samples (No. $1-3$ of Table 1) do not allow a correct measurement of the volume moisture content less than $100 \mathrm{~cm}^{3} / \mathrm{m}^{3}$. It should be noted that in all the moisture meters examined, the problem of «varietal uncertainty» is not solved, since it is necessary to pre-calibrate them.

The existing algorithms for solving the problem of «varietal uncertainty» of dielcometric moisture meters are considered in [1-5]. These include the following algorithms.

A. Algorithm with the introduction of dielectric cylinders into the working space.

$B$. Algorithms: with the separation of flows of the investigated emulsion; with the addition of a predetermined water flow rate; with additives of water and controlled medium.

$C$. Algorithm with the use of least squares methods.

$D$. Algorithm with the use of polynomials Lagrange.

$E$. Algorithms of additive and multiplicative types.

$F$. Additive algorithm with the introduction of a metal plate into the interelectrode space of a capacitivetype capacitor.

The analysis showed that these algorithms are not applicable for increasing the sensitivity of dielcometric moisture meters to values of the volumetric moisture content of the order of $10 \mathrm{~cm}^{3} / \mathrm{m}^{3}\left(10^{-5}\right.$ in dimensionless quantities) for the following reasons: parasitic parameters of the capacitive-type capacitor type (and edge effects for algorithm $F$ ) ; The relative error in measuring the dielectric constant (algorithm $A$ ), the flow and volume of liquids (algorithms $B-E$ ), and the geometric dimensions (algorithm $F$ ) of no more than $10^{-6}$ in the laboratory is an extremely difficult practical task.

On the basis of the foregoing, the way of realization of the dielcometric resonance method with increased sensitivity and simultaneous solution of the problem of «varietal uncertainty» is determined. This is the use of algorithms with a controlled change in the state of the emulsion for the resonance dielcometric method, taking into account the effect on the metrological characteristics of the parasitic parameters and the edge effects of the capacitive-type MT.

The goal of the work is the development, investigation and justification of multifrequency algorithms for determining the moisture content of emulsions of the «nonpolar dielectric-water» type by the method of resonance dielcometry with improved metrological characteristics and solving the problem of «varietal uncertainty».

Proceeding from this goal, the tasks of the work are: determining the areas of applicability of the physical model of the emulsion in the frequency domain; Development of multifrequency algorithms for solving the problem of «varietal uncertainty»; determination of the main metrological characteristics of the proposed 
algorithms, taking into account the influence of parasitic parameters of MT and measuring generator (MG); experimental verification of the correctness of the proposed test algorithms.

Selection of the emulsion model and determination of its applicability areas. As a mathematical model of the emulsion, a model was chosen [7], in which individual water droplets were replaced by ideally conducting spheres of small diameter, which are much larger than their diameter and uniformly distributed throughout the volume. By definition, the volume moisture content is the ratio of the volume of water in the emulsion $V_{\text {wat }}$ to the total emulsion volume $V_{\text {eml }}$ :

$$
W=V_{\text {wat }} / V_{\text {eml }} \text {. }
$$

The mathematical model of the emulsion determines the functional relationship between the values of the volumetric moisture content $W$ and the permittivity $\varepsilon_{e m l}$ and $\varepsilon_{n l d}[7]$ :

$$
\varepsilon_{\text {eml }}=\varepsilon_{\text {nld }}(1+3 W) .
$$

In [7] the frequency domain of the correctness of this model for the HF frequency band was determined, which extends to $2 \mathrm{MHz}$. In this frequency range, water behaves like an ideal conductor with a permittivity equal to infinity.

On the other hand, the electrophysical properties of water are such that in the frequency range from about 100 $\mathrm{MHz}$ to $1 \mathrm{GHz} \varepsilon_{\text {wat }}$ varies insignificantly (from 78.2 to 78.0). In this range, water behaves like a perfect dielectric (the magnitude of the dielectric loss tangent does not exceed 0.005) [8]. Taking into account that $\varepsilon_{\text {wat }} \gg \varepsilon_{\text {eml }}$, we can extend this mathematical model of the emulsion to the frequency range $100 \mathrm{MHz}-1 \mathrm{GHz}$. As a result, a systematic error arises due to the properties of the most simplified mathematical model at these frequencies. Ideally conducting spheres simulating real water droplets in the emulsion $\left(\varepsilon_{w a t}=81\right)$ are replaced in the mathematical model of emulsion by spheres of similar dimensions from an ideal dielectric $\left(\varepsilon_{\text {wat }}=\infty\right)$.

An idealized calculation scheme for determining the systematic error of a mathematical model due to the effect of a change in $\varepsilon_{w a t}$ for a generalized capacitive MT scheme is shown in Fig. 1.

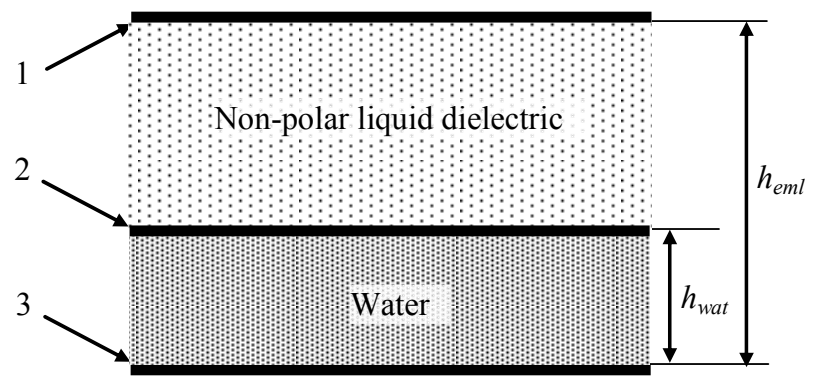

Fig. 1. Idealized calculation scheme of the MT for determining the systematic errors of the emulsion model:

1, 3 - MT electrodes; 2 - flat interface;

$h_{e m l}$ and $h_{w a t}-$ Thicknesses of emulsion and water layers

$$
\left(W=h_{\text {wat }} / h_{\text {eml }}\right)
$$

Water droplets with total volume $V_{\text {wat }}$ are replaced by a linear layer of constant thickness $h_{\text {wat }}$ on the surface of the lower electrode of the MT.

Determination of the value of the systematic error for the $100 \mathrm{MHz}-1 \mathrm{GHz}$ range is reduced to a comparison of capacitance values of a flat capacitor formed by plates 1, 3 of MT for values $\varepsilon_{\text {wat }}=\infty$ и $\varepsilon_{\text {wat }}=81$ of the medium between interface 2 and the lower plate. Taking into account the expression (1) and the fact that the capacitance of the MT between the plates 1 and 3 is determined by the capacity of two series-connected capacitors (with electrodes $1-2$ and electrodes $2-3$ ), the effective dielectric permittivity $\varepsilon^{\prime}$ of the layered medium is generally:

$$
\varepsilon^{\prime}=\frac{1}{\frac{1}{\varepsilon_{\text {wat. }}}+\frac{1-W}{\varepsilon_{\text {nld }}}} .
$$

In Table 2 shows the calculated values of $\varepsilon^{\prime}$ for $\varepsilon_{\text {wat }}=$ $\infty$ и $\varepsilon_{\text {wat }}=81$ for different values of the volumetric moisture content, as well as the values of the systematic errors of the mathematical model in the frequency range $100 \mathrm{MHz}-1 \mathrm{GHz}$, which are defined as $\left(\varepsilon_{\infty}^{\prime}-\varepsilon^{\prime}{ }_{81}\right) / \varepsilon_{\infty}^{\prime}$ $\left(\varepsilon_{\infty}^{\prime} \mathrm{ND} \varepsilon_{81}^{\prime}\right.$ are the equivalent dielectric permittivities of a layered medium for $\varepsilon_{w a t}=\infty, \varepsilon_{w a t}=81$ and $\varepsilon_{n l d}=2$ ).

Table 2

Systematic errors of the mathematical model of the emulsion for the frequency range $100 \mathrm{MГц}-1 \Gamma \Gamma ц$

\begin{tabular}{|c|c|c|c|c|c|}
\hline$W, \%$ & $10^{-1}$ & $10^{-2}$ & $10^{-3}$ & $10^{-4}$ & $10^{-5}$ \\
\hline$\varepsilon_{\infty}^{\prime}$ & 2.22 & 2.02 & 2.002 & 2.0002 & 2.00002 \\
\hline$\varepsilon_{81}^{\prime}$ & 2.162 & 1.97 & 1.953 & 1.9519 & 1.9518 \\
\hline$\frac{\varepsilon_{\infty}^{\prime}-\varepsilon_{81}^{\prime}}{\varepsilon^{\prime} \infty}$ & 0.0261 & 0.0248 & 0.0245 & 0.0241 & 0.0241 \\
\hline
\end{tabular}

Taking into account the linearity of the relation (2), replacing $\varepsilon_{\text {wat }}=81$ by $\varepsilon_{\text {wat }}=\infty$ for a moisture content of $10^{-5} \leq W \leq 0.1$ leads to the appearance of a systematic relative error in determining the $\varepsilon_{e m l}$ of the given mathematical model of the emulsion no more than $2.61 \%$ for the frequency range from $100 \mathrm{MHz}$ to $1 \mathrm{GHz}$, which meets the requirements of practical application.

The general algorithm for solving the problem of «varietal uncertainty». To solve the problem of «varietal uncertainty» and take into account the influence of parasitic capacitances $\mathrm{MT}$ and $\mathrm{MG}$, a number of multifrequency algorithms are proposed, the generalized form of which is the four-frequency algorithm. Fig. 2 shows a generalized equivalent circuit for the replacement of the resonant moisture meter, which characterizes capacitive MT of both concentrated and distributed type. 


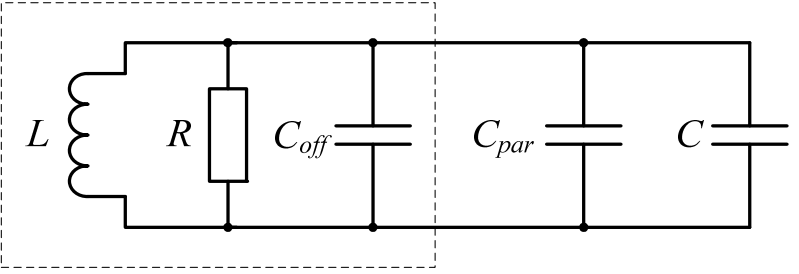

Measuring generator

Fig. 2. Generalized equivalent circuit of the replacement of a resonant moisture meter with a capacitive-type MT: $L$ - inductive element of the MG; $C_{\text {off }}$ - parasitic capacitance of the generator; $C_{p a r}-$ «blind» (constructive) capacitance of the

MT; $C$ - working capacity of the measuring transducer; $R$ - loss resistance

Four-frequency algorithm requires the following four stages.

1. Determination of the resonance frequency $F_{\text {off }}$ of the oscillatory circuit with the MT OFF.

2. Determination of the frequency of the resonance $F_{\text {air }}$ of the oscillatory circuit with the connected MT filled with air.

3. Determination of the frequency of the resonance of the $F_{n l d}$ of the oscillatory circuit with connected MT filled with an anhydrous liquid nonpolar dielectric.

4. Determination of the resonance frequency $F_{\mathrm{eml}}$ of the oscillating circuit with the connected MT filled with the emulsion under investigation.

Four values of the resonant frequencies of the oscillatory circuit make it possible to determine four unknown quantities. Depending on how the measuring transducer is filled, the value $C$ takes the following different values. For the case of MT filling with air, this value is equal to the working capacitance of the MT in air $C=C_{a i r}$; for the case of MT filling with a dehydrated liquid nonpolar dielectric $C=\varepsilon_{n l d} \cdot C_{\text {air }}$; for the case of filling the API with the emulsion investigated $C=\varepsilon_{e m l}$. $C_{\text {air }}$.

The value of the «blind» capacitance $C_{p a r}$ is constant and is determined by the constructive method of fastening the potential electrode MT. In general, the value of $C_{p a r}$ is the fraction $m$ from the working capacity of the measuring transducer $C_{a i r}$ :

$$
C_{\text {par }}=m \cdot C_{\text {air }} ;(m \geq 0) .
$$

For simplicity, we will assume that the value $m$ is given, and the reduced $R L C$ oscillatory circuit is a lossless loop $(R=\infty)$.

The practical implementation of this multifrequency algorithm for determining the values of $\varepsilon_{n l d}$ and $\varepsilon_{e m l}$ consists in the sequential determination of the resonant frequencies the $F_{\text {off }}, F_{\text {air }}, F_{\text {nld }}, F_{\text {eml }}$. of the circuits. Here, the frequency $F_{\text {off }}$ is the resonance frequency of the circuit $L, C_{\text {off }}$ with the MT OFF:

$$
F_{\text {off }}=\frac{1}{2 \pi \sqrt{L C_{o f f}}} .
$$

The next step is to determine the resonance frequency $F_{\text {air }}$ of the circuit at the MT filled with air:

$$
F_{\text {air }}=\frac{1}{2 \pi \sqrt{L C_{\text {off }}+L C_{\text {air }}(1+m)}} .
$$

When the MT is filled with a dehydrated liquid nonpolar dielectric, the resonance frequency $F_{n l d}$ is:

$$
F_{n l d}=\frac{1}{2 \pi \sqrt{L C_{o f f}+L C_{\text {air }} m+L C_{\text {air }} \varepsilon_{\text {nld }}}} .
$$

And, finally, when the MT is filled with the emulsion investigated, the resonance frequency $F_{e m l}$ is:

$$
F_{\text {eml }}=\frac{1}{2 \pi \sqrt{L C_{o f f}+L C_{\text {air }} m+L C_{\text {air }} \varepsilon_{\text {eml }}}} .
$$

The solution of the system of equations defined by expressions (5) - (8) with respect to unknown quantities $\varepsilon_{n l d}$ и $\varepsilon_{e m l}$ allows to determine the unknown moisture content $W$ through the values of the resonance frequencies $F_{\text {off }}, F_{\text {air }}, F_{n l d}, F_{\text {eml }}$ and the constructive coefficient of the measuring transducer $m$ :

$$
W=\frac{A}{3 B},
$$

where $A=F_{\text {air }}^{2} \cdot F_{\text {nld }}^{2} \cdot\left(F_{\text {off }}^{2}-F_{\text {eml }}^{2}\right)-F_{\text {air }}^{2} \cdot F_{\text {eml }}^{2} \cdot\left(F_{\text {off }}^{2}-\right.$ $\left.F^{2}{ }_{n l d}\right) ; B=F_{\text {air }}^{2} \cdot F_{\text {eml }}^{2}\left(F_{\text {off }}^{2}-F_{n l d}^{2}\right)-F_{n l d}^{2} \cdot F_{\text {eml }}^{2} \cdot\left(F_{\text {off }}^{2}-F_{\text {air }}^{2}\right) \cdot m$ $/(m+1)$.

Expression (9) is the main generalized metrological characteristic of the four-frequency algorithm for determining moisture content by the method of resonance dielcometry to determine the moisture content of the emulsion as a function of the quantities $F_{o f f}, F_{\text {air }}, F_{n l d}, F_{\text {eml }}$ и $m$.

In the general case, the error in determining the moisture content includes both systematic and random errors. The systematic errors caused by the inaccuracy of the mathematical model of the emulsion were determined earlier.

Based on the analysis of expression (9), the value of the random error in determining the moisture content $\Delta W / W$ as a function of the absolute errors $\left(\Delta F_{\text {off }}, \Delta F_{\text {air }}\right.$, $\left.\Delta F_{\text {nld }}, \Delta F_{\text {eml }} ; \Delta m\right)$ of determination of values $F_{\text {off }}, F_{\text {air }}, F_{\text {nld }}$, $F_{e m l}$ and $m$ has the following form (in this case the processes of determining $F_{o f f}, F_{a i r}, F_{n l d}, F_{e m l}$ and $m$ are independent):

$$
\frac{\Delta W}{W}=\frac{\sqrt{\sum_{n=1}^{5} Y_{n}^{2}}}{W\left(F_{o f f}, F_{\text {air }}, F_{n l d}, F_{\text {eml }}, m\right)},
$$

where

$$
\begin{aligned}
& Y_{1}^{2}=\left(\frac{\partial W}{\partial F_{\text {off }}}\right)^{2} \Delta F_{\text {off }}^{2} ; Y_{2}^{2}=\left(\frac{\partial W}{\partial F_{\text {air }}}\right)^{2} \Delta F_{\text {air }}^{2} \\
& Y_{3}^{2}=\left(\frac{\partial W}{\partial F_{\text {nld }}}\right)^{2} \Delta F_{n l d}^{2} ; Y_{4}^{2}=\left(\frac{\partial W}{\partial F_{\text {eml }}}\right)^{2} \Delta F_{\text {eml }}^{2} ;
\end{aligned}
$$




$$
Y_{5}^{2}=\left(\frac{\partial W}{\partial m}\right)^{2} \Delta m^{2} .
$$

The values of the absolute errors $\Delta F_{\text {off }}, \Delta F_{\text {air }}, \Delta F_{\text {nld }}$, $\Delta F_{e m l}$ and $\Delta m$ include both systematic and random errors in their determination.

The three-frequency algorithm. For $m=0$, the «blind» capacitance of the MT is not taken into account in expression (9). Then expression (9) is essentially simplified and goes over to a form analogous to the expression obtained earlier for $W$ in [8]:

$$
W=\frac{1}{3} \cdot \frac{\frac{1}{F_{e m l}^{2}}-\frac{1}{F_{n l d}^{2}}}{\frac{1}{F_{\text {nld }}^{2}}-\frac{1}{F_{\text {off }}^{2}}} .
$$

Expression (11) is a metrological characteristic of the three-frequency algorithm for determining moisture content by the method of resonance dielcometry.

For the specific design of the capacitive MT [9], the values of the volumetric moisture content $W$ were determined from the exact formula (9) $\left(W_{(9)}\right)$ and the approximate formula $(11)\left(W_{(11)}\right)$ and were $W_{(9)}=0.0215$ и $W_{(11)}=0.0209$, respectively.

The difference between $W_{(9)}$ and $W_{(11)}$ determines the magnitude of the absolute systematic error in determining the moisture content without taking into account the «blind» capacity, and the quantity $\left(W_{(9)}\right.$ и $\left.W_{(11)}\right) / W_{(9)}$ characterizes the systematic relative error. This relative systematic error for $m=0.0664$ and for the MT frequency range up to $2 \mathrm{MHz}$ is: $\left(W_{(9)}-W_{(10)}\right) / W_{(9)}=0.027$ [9].

Analysis of the limiting characteristics of fourand three-frequency algorithms. For a specific moisture meter with MT of lumped type [9], in which four- and three-frequency algorithms are implemented, an analysis was made of the accuracy of determining the moisture content in an emulsion of the «transformer oil-water» type at $F_{\text {off }}=1145290.4 \mathrm{~Hz} ; \Delta F_{\text {off }}=2 \mathrm{~Hz} ; F_{\text {air }}=946516.0 \mathrm{~Hz}$; $\Delta F_{\text {air }}=1.5 \mathrm{~Hz} ; F_{\text {nld }}=827303.9 \mathrm{~Hz} ; \Delta F_{\text {nld }}=0.9 \mathrm{~Hz}$; $F_{\text {eml }}=821291.0 \mathrm{~Hz} ; \Delta F_{\text {eml }}=3.4 \mathrm{~Hz} ; m=0.0664$; $\Delta m=0.0038$. The analysis was made for the fourfrequency algorithm, as the most accurate one.

The values of $m$ and $\Delta m$ were determined by calculation, proceeding from the geometrical dimensions of the MT and the dielectric characteristics of the fluoroplastic-4: a structural insulating element that fixes the potential electrode of the MT and determines the value of the «blind» capacitance of the MT [9].

The calculated value of the relative error in determining the moisture content obtained from expressions $(5)-(10)$ was: $\Delta W / W=0.011$. This value defines the restriction on the lower limit of the measured moisture content to moisture meters with a concentrated type of MT for four- and three-frequency algorithms (in the $\mathrm{HF}$ range up to $2 \mathrm{MHz}$ ) of the order of $600 \mathrm{~cm}^{3} / \mathrm{m}^{3}$ [9].
Two-frequency algorithm. The analysis of expressions $(9,10)$ and the circuit in Fig. 2 showed that an increase in sensitivity is facilitated by a decrease in the effect of capacitance $C_{\text {off }}$ on the resonance frequency of the MT. The minimum influence of the parasitic capacitance of the generator will be at $C_{\text {off }}=0$. In this case, $m=0, \Delta m=0, F_{\text {off }}=\infty$, and expression (11) is transformed to the form:

$$
W=\frac{1}{3} \cdot \frac{\frac{1}{F_{\text {eml }}^{2}}-\frac{1}{F_{\text {nld }}^{2}}}{\frac{1}{F_{\text {nld }}^{2}}}=\frac{1}{3} \cdot \frac{F_{\text {nld }}^{2}-F_{\text {eml }}^{2}}{F_{\text {eml }}^{2}} .
$$

For small values of the relation

$$
\left(F_{\text {nld }}^{2}-F_{\text {eml }}^{2}\right) / F_{\text {eml }}^{2}<0.1
$$

the expression (12) is simplified and has the following form, up to terms of the second order:

$$
W \approx \frac{2}{3} \cdot \frac{F_{n l d}-F_{e m l}}{F_{\text {eml }}} .
$$

Expressions (12) and (13) are a metrological characteristic of the two-frequency algorithm for determining moisture content by the method of resonance dielcometry.

Technical aspects of practical realizability of the two-frequency algorithm. Let's analyze the technical possibilities of creating a MT with the values $m=0$ and $C_{\text {off }}=0$. The most radical way to ensure $m=0$ is to replace the structural dielectric material that supports the potential electrode of the capacitive MT with a «metallic» insulator, for example, the implementation of the MT in the form of a short-circuited quarter-wave line. In this case, the central (potential) electrode of this line is tightly connected to the outer electrode, and the liquid under investigation is in the space between these electrodes [10]. An MT of this type is a system with distributed parameters and allows the mode $m=0$ to be realized. As shown in $[6,10]$, the transition to the microwave frequency range makes it possible to substantially increase the sensitivity of the resonance dielcometric method. The main problem in this case is the large geometric length of the MT when operating in the frequency range $100 \mathrm{MHz}-1 \mathrm{GHz}$, as well as the small difference between the first and subsequent resonant frequencies of distributed type MT.

The distributed type IP with the measuring element in the form of a stepped inhomogeneous coaxial resonator (SICR), proposed and investigated in [10], is free from the indicated shortcomings and allowed to realize its operation in the microwave frequency range (100-200 $\mathrm{MHz}$ ) with its minimum dimensions.

In order to realize the case $C_{\text {off }}=0$, a characteristic of a SICR-based MT was used, consisting in an essentially larger value of its quality factor with respect to capacitive foci of a concentrated type. This makes it possible to realize the $C_{o f f}=0$ mode due to the very small coupling factor of the measuring oscillator with the PI via 
an inductive loop connection, which is geometrically located near the grounded end of the internal (potential) electrode of the SICR. At the same time, a minimal influence of the coupling loop on the electric field in the SICR is ensured, since the electric field in this place of the SICR is extremely small, and the introduced capacitance of the IG in the working capacitance of the SICR is substantially reduced and its influence can be neglected. Thus, a two-frequency algorithm for resonance dielcometry $\left(m=0, C_{o f f}=0\right)$ is realized in moisture content meters of the SICR type. This algorithm is the theoretical limit of resonance dielcometry, when two unknown quantities $\left(\varepsilon_{e m l}\right.$ and $\left.\varepsilon_{n l d}\right)$ and moisture content, as a function of these quantities, are determined by two values of the measured frequencies.

Results of experimental investigations. For experimental verification of the results of theoretical studies, the moisture content of the prepared test emulsions of the «transformer oil-water» type was determined by two specific moisture meters: with lumped type MT [9] and a distributed type MT in the form of SICR [10]. Experimental studies of the first moisture meter were carried out to test the generalized fourfrequency algorithm, and the purpose of the second moisture meter was to test the two-frequency algorithm. The results are shown in Table. 3 .

Table 3

The results of the experimental determination of the volume moisture content of the test emulsions

\begin{tabular}{|c|c|c|c|c|}
\hline \multirow[b]{2}{*}{ MT type } & \multicolumn{2}{|c|}{$\begin{array}{l}\text { Moisture content, } \\
\mathrm{cm}^{3} / \mathrm{m}^{3}\end{array}$} & \multirow{2}{*}{$\begin{array}{c}\text { Absolute } \\
\text { discrepancy, } \\
\mathrm{cm}^{3} / \mathrm{m}^{3}\end{array}$} & \multirow{2}{*}{$\begin{array}{c}\text { Relative } \\
\text { discrepancy, } \\
\%\end{array}$} \\
\hline & $\begin{array}{c}\text { Prepared } \\
\text { test } \\
\text { emulsion }\end{array}$ & $\begin{array}{c}\text { Measured } \\
\text { value }\end{array}$ & & \\
\hline $\begin{array}{l}\text { Concentrated } \\
\text { MT, HF range } \\
(1 \mathrm{MHz})\end{array}$ & $\begin{array}{c}5000 \pm \\
20\end{array}$ & $4800+54$ & \pm 200 & \pm 4.0 \\
\hline \multirow{2}{*}{$\begin{array}{l}\text { Distributed IP } \\
\text { CSCR, } \\
\text { microwave } \\
\text { range } \\
(0.1 \mathrm{GHz})\end{array}$} & $100 \pm 5.1$ & $98.3 \pm 0.6$ & \pm 1.7 & \pm 1.8 \\
\hline & $10 \pm 1$ & $9.51 \pm 0.5$ & \pm 0.49 & \pm 5.2 \\
\hline
\end{tabular}

For a moisture meter with concentrated type of MT, the values of the volumetric moisture content were determined in accordance with the expression (9), and for a moisture meter with distributed type of MT - in accordance with the expression (12), absolute errors were determined using expression (10) for both moisture meters.

The correctness of the results of theoretical studies characterizes the magnitude of the absolute and relative discrepancies between the measured values of the volumetric moisture content and the moisture content of the prepared test emulsions. In this case, the magnitude of the absolute divergence was determined as the difference between the moisture content of the prepared test emulsion and its calculated value, and the magnitude of the relative divergence as the ratio of the absolute error to the moisture content of the prepared test emulsion.

Analysis of the data given in Table 3 shows the following.

1. The values of the relative discrepancies do not exceed $4 \%$ and $5.2 \%$ (for the concentrated and distributed types of MT, respectively), which indicates a good coincidence of the calculated results with the moisture content of the test emulsions and the correctness of the results of the theoretical studies.

2. The magnitude of the absolute divergence for a moisture analyzer with concentrated type of MT (200 $\mathrm{cm}^{3} / \mathrm{m}^{3}$ ) practically confirms the results of estimating the lower limit of the measured volumetric moisture content $\left(600 \mathrm{~cm}^{3} / \mathrm{m}^{3}\right)$.

3. A moisture meter with distributed type MT in the form of SICR makes it possible to measure the moisture content of emulsions based on nonpolar liquid dielectrics with lower limit of $10 \mathrm{~cm}^{3} / \mathrm{m}^{3}$ without preliminary calibration operations.

4. Metrological characteristics of a moisture meter with distributed type of MT in the form of SICR exceed the analogous characteristics of the known dielcometric moisture meters presented in Table 1 .

\section{Conclusions.}

The proposed and practically realized multifrequency algorithms for determining the volumetric moisture content of liquid emulsions by the method of resonance dielcometry, taking into account parasitic parameters of MT and MG, solved the problem of «varietal uncertainty» of the liquids under investigation, significantly improving the metrological characteristics of the dielcometric method, and measuring the volume moisture content of liquid nonpolar dielectrics up to $10^{-5}$.

The correctness of the results of theoretical investigations is confirmed experimentally with a maximum value of the relative discrepancy of not more than $5.2 \%$.

A promising direction for the further development of the proposed multifrequency algorithms in order to improve the metrological characteristics can be the consideration of their systematic errors.

\section{REFERENCES}

1. Golub E.Yu. Metody i sredstva povyshenija tochnosti diel'kometricheskih vlagomerov. Diss. cand. techn. nauk [Methods and means of increasing the accuracy of dielectric metric moisture meters. Cand. tech. sci. diss.]. Kharkov, 2016. 199 p. (Rus).

2. Zabolotnyy A.V. Technical realization of additive tests in a capacitive primary converter of the diesel-electric moisture meter of petroleum products. Measuring and Computing Devices in Technological Processes, 2013, no.3, pp. 49-53. (Rus). 
3. Marcel L. Mounge. Method and apparatus for measuring and calculating bulk water in crude oil. Patent USA, no.4916940, 1990.

4. Zabolotnyy O.V. Sposib vymiryuvannya volohosti materialiv [The method of measuring moisture content]. Patent UA, no.201201992, 2014. (Ukr).

5. Platon G., Ramm C., Lohr K. Capacitive moisture sensor. Patent USA, no.7129713, 2006.

6. Suslin M. A. Mikrovolnovyy kontrol' aviatsionnykh GSM s ispol'zovaniyem radiotekhnicheskikh metodov rascheta tsepey $s$ raspredelennymi parametrami [Microwave monitoring of aviation fuel and lubricants using radio engineering methods for computing circuits with distributed parameters]. Moscow, Mashinostroenie Publ., 2006. 120 p. (Rus).

7. Rudakov V.V., Korobko A.I., Korobko A.A. Electrophysical model of behavior emulsion mineral oil - water engineering type. Bulletin of NTU «KhPI», 2009, no.39, pp. 158-161. (Rus). 8. Hippel A.R. Dielektriki $i$ ikh primeneniye [Dielectrics and their application]. Moscow, Gosenergoizdat Publ., 1953. 336 p. (Rus).

How to cite this article:

Korobko A.A. Multifrequency algorithms for determining the moisture content of liquid emulsions by the method of resonance dielcometry. Electrical engineering \& electromechanics, 2017, no.3, pp. 40-46. doi: 10.20998/2074272X.2017.3.06.
9. Rudakov V.V., Korobko A.A. Increasing the sensitivity of the moisture content measurements in transformer oil dielcometric method in resonant mode. Bulletin of $N T U$ «KhPI», 2014, no.50(1092), pp. 143-149. (Rus).

10. Rudakov V.V., Korobko A.A. A high sensitive microwave measuring device of the moisture content in the non-polar dielectric liquids based on an inhomogeneous step coaxial resonator. Electrical engineering \& electromechanics, 2016, no.5, pp. 51-56. (Rus). doi: 10.20998/2074-272X.2016.5.08.

\section{Received 13.04.2017}

A.A. Korobko, Postgraduate Student,

National Technical University «Kharkiv Polytechnic Institute», 2, Kyrpychova Str., Kharkiv, 61002, Ukraine, phone +3809365080 88,

e-mail: andarleks@gmail.com 